\title{
Associating liver partition and portal vein ligation for staged hepatectomy versus sequential transarterial chemoembolization and portal vein embolization in staged hepatectomy for HBV- related hepatocellular carcinoma: a randomized comparative study
}

\author{
Peng-Peng Li ${ }^{1 \#}$, Gang Huang ${ }^{1 \#}$, Ning-Yang Jia ${ }^{1}$, Ze-Ya Pan ${ }^{1}$, Hui Liu ${ }^{1}$, Yun Yang ${ }^{1}$, Cheng-Jian He ${ }^{1}$, \\ Wan Yee Lau ${ }^{2}$, Ye-Fa Yang ${ }^{1}$, Wei-Ping Zhou ${ }^{1}$ \\ ${ }^{1}$ Eastern Hepatobiliary Surgery Hospital, Shanghai, China; ${ }^{2}$ Faculty of Medicine, The Chinese University of Hong Kong, Prince of Wales Hospital, \\ Hong Kong, China \\ Contributions: (I) Conception and design: PP Li; (II) Administrative support: WP Zhou, YF Yang; (III) Provision of study materials or patients: PP Li, \\ G Huang, NY Jia, ZY Pan, H Liu, Y Yang, CJ He, YF Yang, WP Zhou; (IV) Collection and assembly of data: H Liu, Y Yang; (V) Data analysis and \\ interpretation: Y Yang, H Liu; (VI) Manuscript writing: All authors; (VII) Final approval of manuscript: All authors. \\ \#These authors contributed equally to this work. \\ Correspondence to: Wei-Ping Zhou, MD. Eastern Hepatobiliary Surgery Hospital, Second Military Medical University, Shanghai, China; Key \\ Laboratory of Signaling Regulation and Targeting Therapy of Liver Cancer (SMMU), Ministry of Education. Shanghai, China; Shanghai Key \\ Laboratory of Hepatobiliary Tumor Biology (EHBH), Shanghai, China. Email: ehphwp@126.com; Ye-Fa Yang, MD. Eastern Hepatobiliary \\ Surgery Hospital, Second Military Medical University, 225 Changhai Rd., Shanghai, China. Email: yangyefa66@163.com; Wan Yee Lau, MD, \\ FRCS, FACS, FRACS (Hon). Faculty of Medicine, The Chinese University of Hong Kong, Prince of Wales Hospital, Shatin, Hong Kong, China. \\ Email: josephlau@cuhk.edu.hk.
}

Background: Both portal vein embolization (PVE) and associating liver partition and portal vein ligation for staged hepatectomy (ALPPS) have merits and demerits when used in patients with unresectable liver cancers due to insufficient volumes in future liver remnant (FLR).

Methods: This study was a single-center, prospective randomized comparative study. Patients with the diagnosis of hepatitis B related hepatocellular carcinoma (HCC) were randomly assigned in a 1:1 ratio to the 2 groups. The primary endpoints were tumor resection and three-year overall survival (OS) rates.

Results: Between November 2014 to June 2016, 76 patients with unresectable HBV-related HCC due to inadequate volume of FLR were randomly assigned to ALPPS groups $(n=38)$ and TACE + PVE groups $(n=38)$. Thirty-seven patients (97.4\%) in the ALPPS group compared with 25 patients $(65.8 \%)$ in the TACE + PVE group were able to undergo staged hepatectomy (risk ratio $1.48,95 \% \mathrm{CI}: 1.17-1.87, \mathrm{P}<0.001$ ). The threeyear OS rate of the ALPPS group (65.8\%) (95\% CI: 50.7-80.9) was significantly better than the TACE + PVE group (42.1\%) (95\% CI: 26.4-57.8) (HR 0.50, 95\% CI: 0.26-0.98, two-sided P=0.036). However, no significant difference in the OS rates between patients who underwent tumor resection in the 2 groups of patients was found (HR 0.80, 95\% CI: 0.35-1.83, two-sided P=0.595). Major postoperative complications rates after the stage- 2 hepatectomy were $54.1 \%$ in the ALPPS group and $20.0 \%$ in the TACE + PVE group (risk ratio $2.70,95 \% \mathrm{CI}: 1.17-6.25, \mathrm{P}=0.007$ ).

Conclusions: ALPPS resulted in significantly better intermediate-term OS outcomes, at the expenses of a significantly higher perioperative morbidity rate compared with TACE + PVE in patients who had initially unresectable HBV-related HCC.

Keywords: Associating liver partition and portal vein ligation for staged hepatectomy (ALPPS); portal vein embolization (PVE); hepatocellular carcinoma (HCC); resection rate; prognosis 
Submitted Jan 30, 2020. Accepted for publication May 09, 2020.

doi: $10.21037 / \mathrm{hbsn}-20-264$

View this article at: http://dx.doi.org/10.21037/hbsn-20-264

\section{Introduction}

Hepatocellular carcinoma (HCC) accounts for about $80 \%$ of all primary liver cancers (1). Partial hepatectomy is the most commonly used treatment aiming at cure (2). Only $20-30 \%$ of patients with HCC are resectable worldwide (3). Insufficient remnant liver volume and function are the main causes of post-hepatectomy liver failure (PHLF) (4), which is a serious and lethal complication $(4,5)$.

Both portal vein embolization (PVE) proposed by Makuuchi (6), and portal vein ligation (PVL) proposed by Clavien (7) and Kianmanesh (8) aim to induce liver hypertrophy in FLR to allow a staged hepatectomy to be carried out in patients who initially have unresectable liver cancers due to inadequate volumes of FLR. However, both PVE and PVL have the shortcoming that adequate increase in volume of FLR usually takes more than 4 weeks, with some patients 2 to 4 months. During the waiting period, tumors can progress and become unresectable (9). ALPPS, accidentally discovered by Hans Schlitt (10), can more rapidly promote increase in volume of FLR, thus allowing an earlier stage-2 hepatectomy (11). However, the reported postoperative mortality and complication rates of ALPPS are high and the long-term oncological survival outcomes are unclear (12).

Recent reports on associating liver partition and PVL for staged hepatectomy (ALPPS) $(13,14)$ mainly focus on colorectal liver metastases. To our knowledge, there is no randomized comparative studies on ALPPS versus PVE in HCC patients. Therefore, our objective in this trial was to evaluate whether ALPPS gave rise to better resection rates and intermediate-term overall survival (OS) outcomes as compared to TACE + PVE.

\section{Methods}

\section{Study design and ethics}

This was a prospective, randomized, comparative study on patients with hepatitis B virus (HBV)-related HCC treated with either ALPPS or TACE + PVE. Eligible patients were assigned in a 1:1 ratio to the 2 groups.

The study was conducted in accordance with the Declaration of Helsinki (as revised in 2013). The study was approved by the Ethics Committee of the Eastern Hepatobiliary Hospital (approval no. EHBHKY2014-03-019). A written informed consent was obtained from all the patients for their data to be used for research. The study is registered in the Chinese Clinical Trials Registry (registration number ChiCTRIOC-14005646).

\section{Patients}

All the patients were enrolled in Eastern Hepatobiliary Surgery Hospital. Eligibility criteria were patients with: (I) age 18 to 75 years. (II) An unresectable HBV-related HCC due to inadequate volume of FLR $(15,16)$. In HCC patients with a background of fibrosis or cirrhosis, a FLR/ SLV ratio $>40 \%$ (FLR/BW $>0.8 \%$ ) was considered safe for a stage-2 hepatectomy $(15,17)$. The SLV was calculated using the formula: $\mathrm{SLV}(\mathrm{mL})=706.2 \times \mathrm{BSA}\left(\mathrm{m}^{2}\right)+2.4$ (18). (III) No history of anti-cancer therapy. (IV) BCLC stage-0, A, and B (19). (V) Child-Pugh liver function Class A and baseline serum alanine aminotransferase (ALT) level $<2$ times the upper limit of normal. (VI) Informed consent from the patient.

The exclusion criteria were patients with: (I) resectable HCC. (II) Unresectable HCC with intra- or extra-hepatic metastases. (III) Severe portal hypertension defined as presence of liver stiffness $>20 \mathrm{kPa}$ on FibroTouch assessment (20), and/or endoscopy showing esophageal varices not flattened by air insufflation (21). (IV) An ASA operative risk $\geq$ III. (V) Refusal to participate in this study.

\section{Procedures}

In the ALPPS group, after the stage- 1 operation, the liver was reexamined once weekly using enhanced CT. The volume of FLR was calculated using the threedimensional visualization technique (the Three-dimensional Visualization Software, Shenzhen XuDong Digital Medical Imaging Technology Co., Ltd or the IQQA-Liver EDDA Technology Inc., Princeton, NJ). If increase in volume of FLR was sufficient, a stage- 2 operation was performed, otherwise the assessment was repeated at a weekly interval until the increase in size of FLR met the predefined 
requirements, or else it was defined as treatment failure. Considering the increase in volume of FLR being slow after PVE, and a long wait could lead to tumor progression, the TACE + PVE group of patients was first treated with TACE. After 2 weeks, when the liver function had returned to normal, PVE was performed (22). The FLR was assessed in the same way as the patients in the ALPPS group. This 4 weekly TACE and assessment of volume of FLR cycle was repeated until the increase in volume of FLR met the predefined requirements for a stage- 2 hepatectomy, or else the treatment was defined as failure. Rescue ALPPS would be recommend to patients in the TACE + PVE group whose FLR failed to increase to the required volume.

Treatment failure was defined as: (I) carcinomatosis or extrahepatic metastasis. (II) Liver kinetic growth rate (KGR) below $2 \% /$ wk at any time. (III) Complications which prevented stage-2 hepatectomy. Treatments such as TACE, sorafenib, or supportive treatment were then offered to these patients.

Preoperatively, all patients underwent upper abdominal intravenous contrast enhanced CT for $3 \mathrm{D}$ reconstruction and liver volume calculation, indocyanine green clearance test (ICG R15), FibroTouch (Healthcare Medical Technology Co., Ltd., Wuxi, China), gastroscopy and ultrasonic examination.

All patients received antiviral therapy using entecavir $(0.5 \mathrm{mg} /$ day $)$ or tenofovir (300 mg/day) before surgery. The antiviral therapy was continued unless there was unacceptable toxicity $(23,24)$.

The KGR was calculated either by the mean volume increase per day from the baseline to the final volume before stage- 2 hepatectomy or by the following formula: $\mathrm{KGR}=$ degree of increase of liver volume (\%)/time elapsed (weeks) from the baseline to the final volume before stage-2 hepatectomy (25).

All surgeries were open hepatectomy by a single surgical team following the previously reported techniques $(14,26)$. For the ALPPS stage-1 operation, the right or left portal vein on the side of liver containing the tumor was ligated. Both partial and complete liver parenchymal transection were carried out. The middle hepatic vein was preserved in the stage- 1 operation. For the ALPPS stage- 2 operation: The portal vein, hepatic artery, bile duct and hepatic veins supplying the parts of the liver containing the tumor were divided. The specimen was removed. The middle hepatic vein was resected in left/right hepatectomy.

All TACE and PVE procedures were performed by a single interventional radiologist team using the previously reported techniques (22). Through a super-selective catheter into the tumor artery, $40 \mathrm{mg}$ of epirubicin, $15 \mathrm{mg}$ of hydroxycamptothecin, $16 \mathrm{~mL}$ of iodized oil, $1 / 3$ of gelatin sponge particles (diameter $560-710 \mu \mathrm{m}$ ) and $1 / 4$ of microspheres (diameter 1,000-1,400 $\mu \mathrm{m}$ ) were injected. When the liver function returned to normal after 2 weeks, PVE was performed under light patient sedation using percutaneous puncture of the contralateral portal vein to the lesion under sonographic guidance. Embolisation was done with gelatin sponges and steel coils.

\section{Outcomes and follow-up}

The primary endpoints were the tumor resection and the three-year survival rates. The secondary endpoints were: (I) the rates of increase in volume of FLR. (II) The time to reach to the predefined FLR volume for the stage-2 hepatectomy. (III) Intraoperative data, and (IV) postoperative mortality and morbidity rates.

Portal hypertension was defined as presence of thrombocytopenia (PLT $<100 \times 10^{9} / \mathrm{L}$ ), with splenomegaly (a splenic length $\geq 12 \mathrm{~cm}$ ) (21), or with gastroesophageal varices on preoperative gastroscopy. Severe liver fibrosis was defined as a liver stiffness $\geq 12.5 \mathrm{kPa}$ (27). The operation time for the stage- 1 operation in TACE + PVE groups is defined as the duration in the PVE procedure. Morbidity was classified according to the Clavien-Dindo classification (28), with major complications as $\geq$ grade IIIa, and severe complications as $\geq$ grade IIIb. Posthepatectomy liver failure (PHLF) was defined and graded using the "ISGLS" criteria (29). The degrees of liver fibrosis were evaluated using the METAVIR scoring (30). Surgical margin was defined as the shortest macroscopic distance from the edge of tumor to the resection plane. R0 resection was defined as non-existence of tumor cells at the plane of transection on microscopic examination.

Patients were followed-up once every month after treatment and re-examined with enhanced CT or MRI, liver function, AFP, HBV-DNA and chest radiography once every three months. Patients diagnosed to have HCC recurrence or metastasis were treated actively, depending on the location and number of recurrent tumor, the liver functional status and the general condition of the patient.

\section{Sample size calculation}

Previous reports showed more than $90 \%$ of patients completed the stage-2 hepatectomy using ALPPS, while 
about $60 \%$ of patients using PVE $(9,11,26)$. Using an alpha risk of 0.05 and a power of $80 \%$, a sample size of 30 patients in each group was required. Considering possible violation of protocol and loss to follow-up, 38 patients were entered into each group.

\section{Randomization}

Randomization was done after the multidisciplinary tumor team, which included radiologists, oncologists, and liver surgeons, had assessed the patient to be suitable for both ALPPS and TACE + PVE. Eligible patients were assigned in a 1:1 ratio to the 2 groups using a computer-generated list of random numbers. A research nurse who was not involved in this study assigned the treatments according to consecutive numbers, which were kept in sealed envelopes. Masking was not adopted for the group assignment since the two treatment procedures were different.

\section{Statistical analysis}

Continuous data were expressed as median (range), and categorical data as a count or ratio. The Mann-Whitney U test was used as the non-parametric test for two independent samples. Categorical variables were tested by the Pearson's chi-square or Fisher exact tests. Correlations were analyzed by the Pearson test. The multiple linear regression analysis was performed to identify significant factors affecting the rate of increase in volume of FLR. The Kaplan-Meier method was used to estimate OS. OS was defined as the time from first treatment to death (all causes). The Logrank test was used to compare survival outcomes between the two groups. The Cox regressions were carried out to examine the association between OS and demographic and other covariates. All computations relied on the standard software (SPSS Statistics v24; IBM, Chicago, IL, USA). A 2 -sided $\mathrm{P}<0.05$ was considered as statistically significant. All data were analyzed on the intention-to-treat basis (ITT).

\section{Role of the funding source}

The funders were not involved in study design, data collection, data analysis, data interpretation, or paper writing. All authors had full access to all the data in the study and had final responsibility for the decision to submit for publication.

\section{Results}

From November 2014 to June 2016, 1,146 patients with HCC underwent treatment at our center, 921 of them underwent liver resection directly, 139 of them were suitable for staged hepatectomy. Sixty-three of 139 patients who refused two staged hepatectomy were treated with TACE + sorafenib. Finally, 76 patients entered into this study. This study was censored on June 1, 2019, because all the patients received at least three-years follow-up. Exclusions after randomization and reasons for them were elaborated in Flow chart (flow chart in Figure 1).

\section{General information and preoperative clinical data of patients}

There were no significant differences in the general and preoperative clinical data between the 2 groups (Table 1).

\section{Treatment results of the ALPPS and TACE + PVE groups (Table 2)}

All the 38 patients in the ALPPS Group completed the stage- 1 operation, and 37 patients $(97.4 \%)$ completed the stage- 2 hepatectomy. Only 1 patient with portal hypertension failed to achieve sufficient increase in volume of FLR to undergo hepatectomy.

In the TACE + PVE group, all the 38 patients underwent the first TACE treatment and PVE. Twenty-five patients $(65.8 \%)$ completed stage-2 hepatectomy. The reasons for failure to proceed to stage- 2 hepatectomy are listed in Table 2. There was a significant difference between the ALPPS and the TACE + PVE groups in the completion rates of stage-2 hepatectomy (risk ratio 1.48, 95\% CI: $1.17-$ $1.87, \mathrm{P}<0.001)$. All the six patients who failed to undergo stage-2 hepatectomy due to insufficient increase in the volume of FLR refused to switch to the ALPPS group and they were treated with TACE+ sorafenib. One patient who refused to undergo stage-2 hepatectomy owing to tumor necrosis after PVE didn't receive further treatment except follow-up every three months. When the TACE + PVE group of patients were further subdivided into the portal hypertension $(n=12)$ and the non-portal hypertension $(n=26)$ subgroups, the stage- 2 hepatectomy rate in the non-portal hypertension subgroup was higher, though insignificantly, than the portal hypertension subgroup $(41.7 \%$ versus $76.9 \%$ respectively, $\mathrm{P}=0.078$ ). 


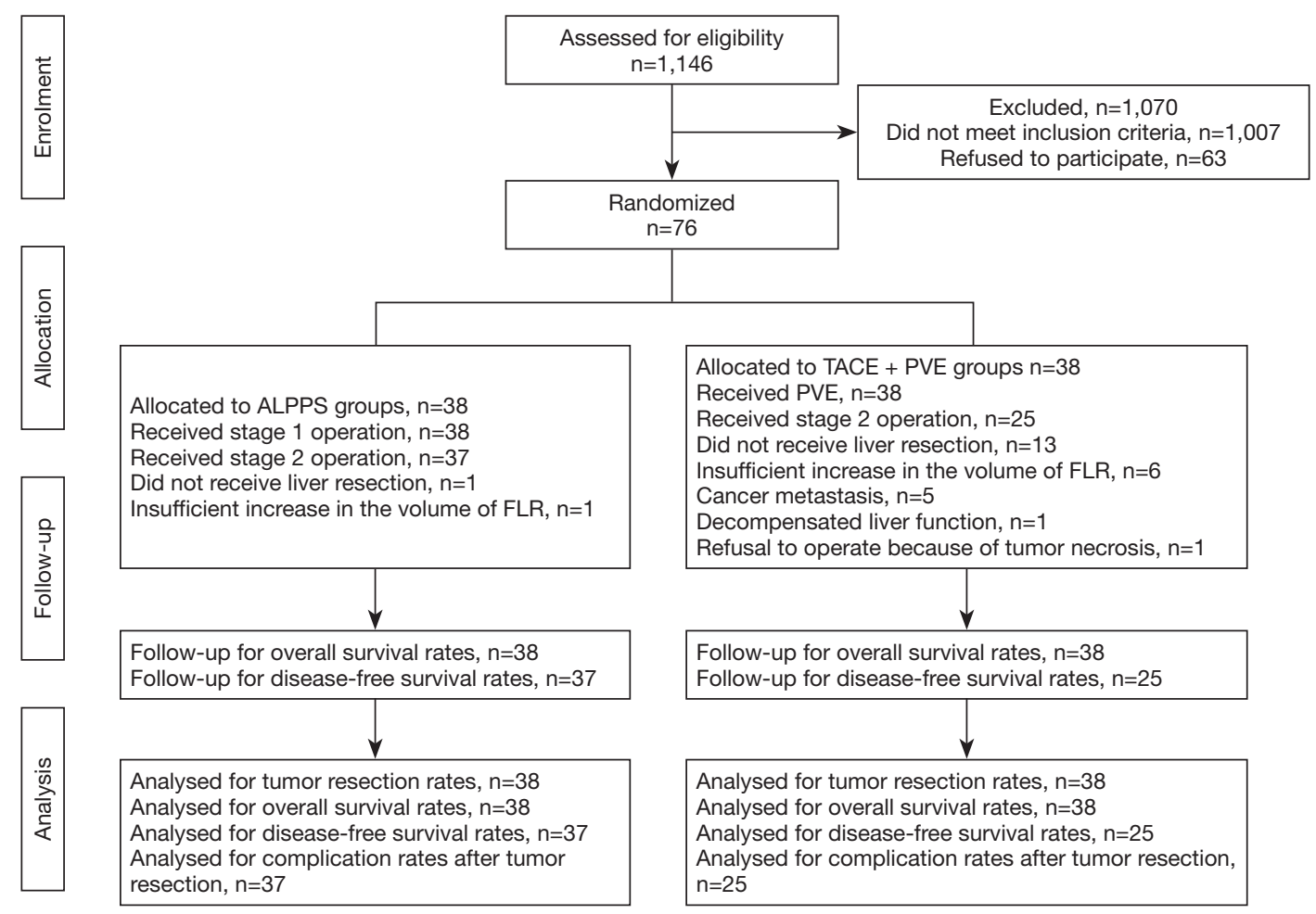

Figure 1 Flow chart of study design. ALPPS, associating liver partition and portal vein ligation for staged hepatectomy; FLR, future liver remnant; TACE, transcatheter arterial chemoembolization; PVE, portal vein embolization.

Table 1 Baseline characteristics of patients in the two groups

\begin{tabular}{|c|c|c|}
\hline Characteristics & \multicolumn{2}{|c|}{ Results } \\
\hline Age (yr) & $48.5(27.0-68.0)$ & $51(32.0-72.0)$ \\
\hline Sex, male, n (\%) & $34(89.5 \%)$ & $32(84.2 \%)$ \\
\hline BMI & $23.7(19.7-31.1)$ & $23.0(20.6-28.6)$ \\
\hline I & $5(13.2 \%)$ & 7 (18.4\%) \\
\hline II & $33(86.8 \%)$ & 31 (81.6\%) \\
\hline III & 0 & 0 \\
\hline \multicolumn{3}{|l|}{ ECOG score, n (\%) } \\
\hline 2 & 0 & 0 \\
\hline HBV-DNA >50 IU/mL, n (\%) & $18(47.4 \%)$ & $21(55.3 \%)$ \\
\hline $\mathrm{HCV}+, \mathrm{n}(\%)$ & 0 & $1(2.6 \%)$ \\
\hline TB $(\mu \mathrm{mol} / \mathrm{L})$ & $13.3(6.7-28.0)$ & $13.2(5.4-31.0)$ \\
\hline
\end{tabular}

Table 1 (Continued) 
Table 1 (Continued)

\begin{tabular}{|c|c|c|}
\hline Characteristics & \multicolumn{2}{|c|}{ Results } \\
\hline ALB $(g / L)$ & $41.5(35.1-51.3)$ & $40.1(33.6-48.7)$ \\
\hline Portal hypertension+, n (\%) & $14(36.8 \%)$ & $12(31.6 \%)$ \\
\hline Gastroesophageal varices, n (\%) & $2(5.3 \%)$ & $2(5.3 \%)$ \\
\hline Spleen diameter (mm) & $101(77.0-140.0)$ & $103(79.0-136.0)$ \\
\hline AFP $>20 \mu g / L, n(\%)$ & $23(60.5 \%)$ & $27(71.1 \%)$ \\
\hline Fibrotouch (kPa) & $8.4(4-21.2)$ & $10.4(5.1-20.3)$ \\
\hline Single tumor, $\mathrm{n}(\%)$ & $19(50 \%)$ & $17(44.7 \%)$ \\
\hline Sum of diameters $(\mathrm{cm})$ & $12(7.0-17.0)$ & $11(5.0-15.0)$ \\
\hline Sum of tumor volume $(\mathrm{mL})$ & $451(18.0-2,493.0)$ & $397(9.0-1,677.0)$ \\
\hline $\mathrm{FLR}_{1} / \mathrm{BW}$ & $0.72 \%(0.39-0.79 \%)$ & $0.65 \%(0.39-0.79 \%)$ \\
\hline \multicolumn{3}{|l|}{ Child-Pugh stage (\%) } \\
\hline A & 100 & 100 \\
\hline 0 & 0 & 0 \\
\hline A & 50 & 44.7 \\
\hline B & 50 & 55.3 \\
\hline
\end{tabular}

The registration number of FDA is K173595. FibroTouch: FibroTouch is a non-invasive detection system for liver fibrosis and has been approved by FDA. BMI, body mass index; TB, total bilirubin; ALT, alanine aminotransferase; ALB, albumin; ASA, American Society of Anesthesiologists; ECOG, Eastern Cooperative Oncology Group; FLR/BW, future liver remnant volume/body weight; BCLC stage, Barcelona Clinic Liver Cancer classification.

\section{Results on increase in volumes of future liver remnant (FLR) and on interstage time intervals (Table 3)}

The daily increase in the volume of FLR $(\mathrm{P}<0.001)$ and the interstage waiting time for stage-2 hepatectomy were significantly better in the ALPPS group $(\mathrm{P}<0.001)$. There was no significant difference between the 2 groups in the final volume of FLR. The changes in tumor volume between the 2 treatment strategies showed no significant difference (ALPPS, $\mathrm{P}=0.408$; TACE + PVE, $\mathrm{P}=0.433$ ).

The differences in the rates of liver hypertrophy in the ALPPS and TACE + PVE groups are shown in Figure $2 A$. The daily volume increase of FLR with the different degrees of liver fibrosis and cirrhosis after ALPPS is shown in
Figure $2 B$. The volume increase of FLR in patients with or without portal hypertension after PVE is shown in Figure 2C.

The results of univariate analysis on significant factors affecting the rate of increase in volume of FLR are shown in Table 4. On multivariate analysis, only the treatment strategy with ALPPS ( $\beta=13.03,95 \%$ CI: $10.03-16.02, \mathrm{P}<0.001)$ and a low FibroTouch elastography value $(\beta=-0.58,95 \%$ CI: -0.92 to $-0.23, \mathrm{P}=0.001$ ) remained as significant factors.

The KGR negatively correlated with the severity of fibrosis and cirrhosis as reflected by the METAVIR scoring in the 37 patients who underwent stage 2 hepatectomy in the ALPPS group ( $\mathrm{P}=0.007)$. The patients in the ALPPS group were then subdivided into the portal hypertension 
Table 2 Intraoperative and postoperative data of patients in the two groups

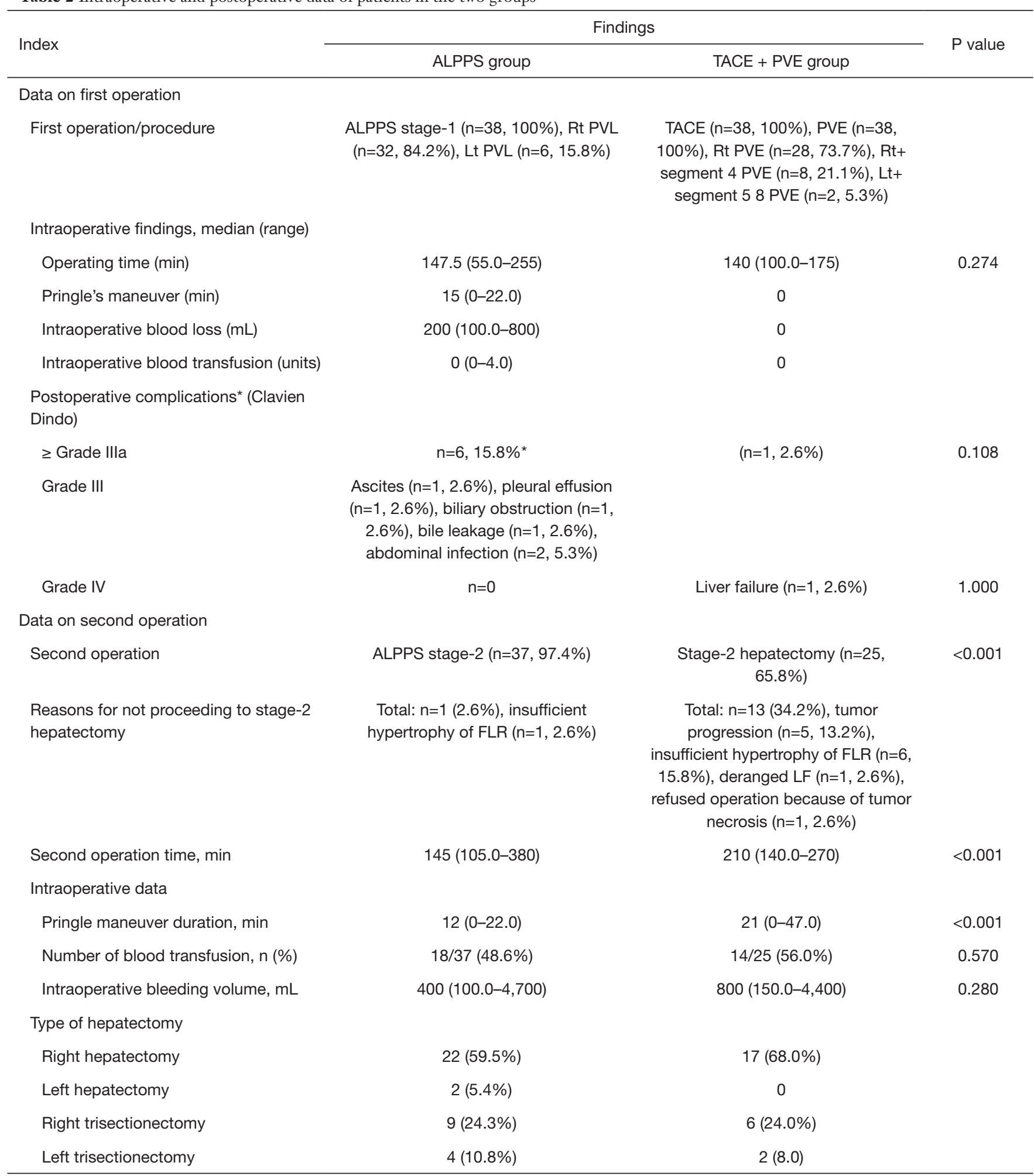

Table 2 (Continude) 
Table 2 (Continude)

\begin{tabular}{|c|c|c|c|}
\hline Index & \multicolumn{2}{|c|}{ Findings } & $P$ value \\
\hline Complications, n (\%) & $35 / 37(94.6 \%)$ & $22 / 25(88 \%)$ & 0.385 \\
\hline Major complications $(\geq \mathrm{IIIa})^{\dagger}$ & 20/37 (54.1\%) & $5 / 25(20 \%)$ & 0.007 \\
\hline Grade III & $n=18,48.6 \%$ & $n=4,16.0 \%$ & \\
\hline Atelectasis + intra-abdominal infection & $1(2.7 \%)$ & 0 & \\
\hline Pleural effusion + hemorrhage & $2(5.4 \%)$ & 0 & \\
\hline Pleural effusion + disruption of wound & $1(2.7 \%)$ & 0 & \\
\hline Bile leakage & $1(2.7 \%)$ & 0 & \\
\hline Grade IV & $\mathrm{n}=2,5.4 \%$ & $n=1,4.0 \%$ & \\
\hline Liver failure & $2(5.4 \%)$ & $1(4.0 \%)$ & \\
\hline Resection margin (cm) & $1(0.5-2.0)$ & $1(0.5-2.2)$ & 0.602 \\
\hline Postoperative liver failure, $\mathrm{n}(\%)$ & $13 / 37(35.1 \%)$ & $6 / 25(24.0 \%)$ & 0.351 \\
\hline Grade A & 5 & 3 & \\
\hline Grade B & 5 & 2 & \\
\hline Rehepatectomy & $1(2.7 \%)$ & $1(4.0 \%)$ & \\
\hline TACE & $2(5.4 \%)$ & $5(20.0 \%)$ & \\
\hline TACE + sorafenib & $5(13.5 \%)$ & 0 & \\
\hline РMCT & $2(5.4 \%)$ & $1(4.0 \%)$ & \\
\hline PMCT + TACE & 0 & $1(4.0 \%)$ & \\
\hline $\mathrm{PMCT}$ + radiotherapy & $2(5.4 \%)$ & 0 & \\
\hline External radiation & $2(5.4 \%)$ & 0 & \\
\hline Sorafenib & $3(8.1 \%)$ & $3(12.0 \%)$ & \\
\hline Supportive treatment & $2(5.4 \%)$ & $2(8.0 \%)$ & \\
\hline
\end{tabular}

*, all complications responded to drainage and conservative treatment; ${ }^{\dagger}$, all complications were coped with conservative treatment, drainage or reoperation. PVL, portal vein ligation; deranged LF, deranged liver function; Lt, left portal trunk; Rt, right portal trunk; PMCT, percutaneous microwave coagulation therapy. 
Table 3 Increase in volume of the future liver remnant in the two groups

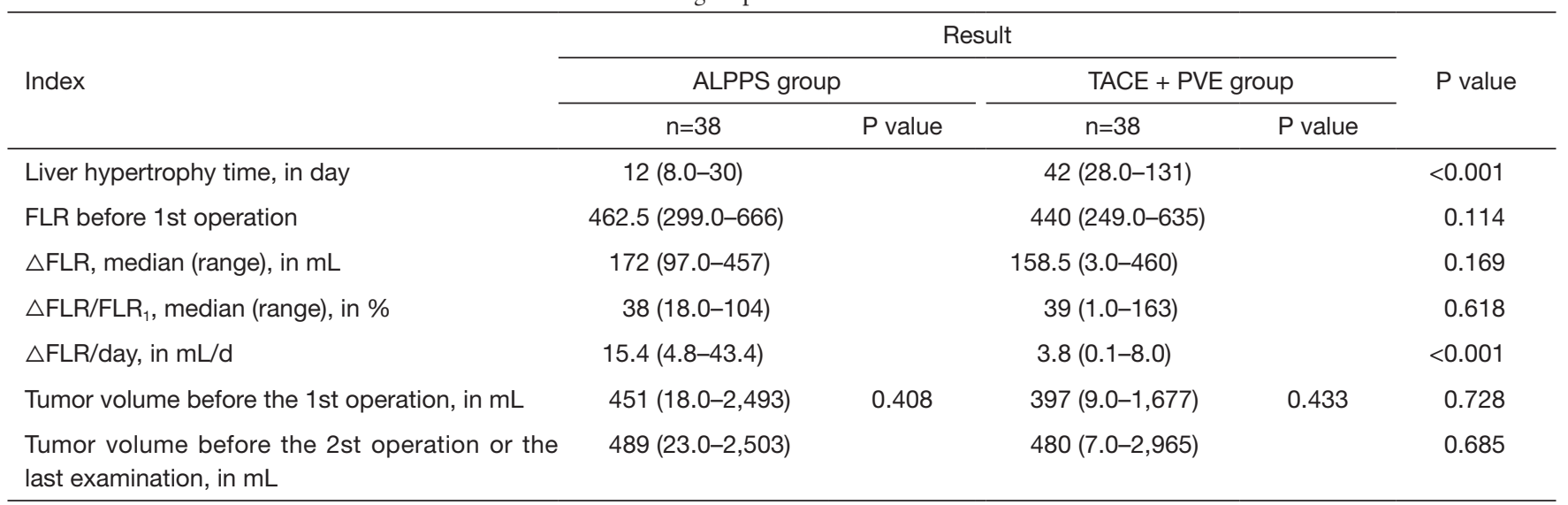

Liver hypertrophy time: the waiting time between the two operations or the time span from the first stage operation to treatment failure. $\triangle F L R$ : the increase in future liver remnant volume between the two operations. $\triangle F L R / F L R 1$ : the increase of future liver remnant volume between the two operations divided by the volume of future liver remnant before the first operation.
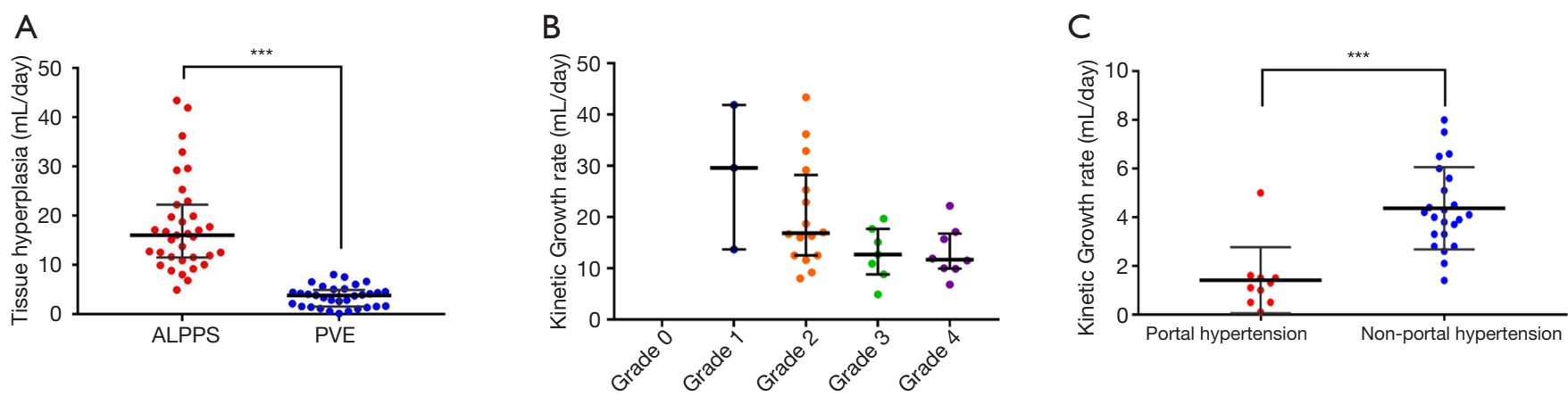

Figure 2 Liver hypertrophies in different groups. (A) The differences in liver hypertrophy in patients of two groups; (B) volume increase of FLR in patients with different severity of fibrosis and cirrhosis after ALPPS; (C) volume increase of FLR in patients with or without portal hypertension after PVE. ***, $\mathrm{P}<0.001$. FLR, future liver remnant; ALPPS, associating liver partition and portal vein ligation for staged hepatectomy; PVE, portal vein embolization.

$(n=14)$ and the non-portal hypertension $(n=24)$ subgroups. There was a significant difference in KGR $(\mathrm{P}=0.022)$, but no significant difference in increase of FLR volume between the 2 subgroups $(\mathrm{P}=0.126)$.

When the patients in the TACE + PVE group were subdivided into the portal hypertension $(n=12)$ and the nonportal hypertension ( $\mathrm{n}=26)$ subgroups, significantly faster KGR $(\mathrm{P}<0.001)$ and larger increase in FLR volume $(\mathrm{P}=0.001)$ were found in the non-portal hypertension subgroup.

\section{Operations and intraoperative findings}

The intraoperative data of the two groups of patients, including the stage-1 operation/procedure, and the stage-2 hepatectomy, are listed in Table 2. Most of the patients in two groups underwent right hepatectomy or trisectionectomy, only 2 patients in the ALPPS groups, whose left liver is larger than right liver, underwent left hepatectomy. The second operation time and Pringle's maneuver time was significantly longer in the TACE + PVE groups than the ALPPS groups. Patients in the TACE + PVE groups undergone more intraoperative bleeding than the patients in the ALPPS groups, but without statistical significance.

\section{Postoperative results}

The postoperative complications after the stage-1 operation/ 
Table 4 Uni- and multi-variate analyses for the increase in volume of the future liver remnants

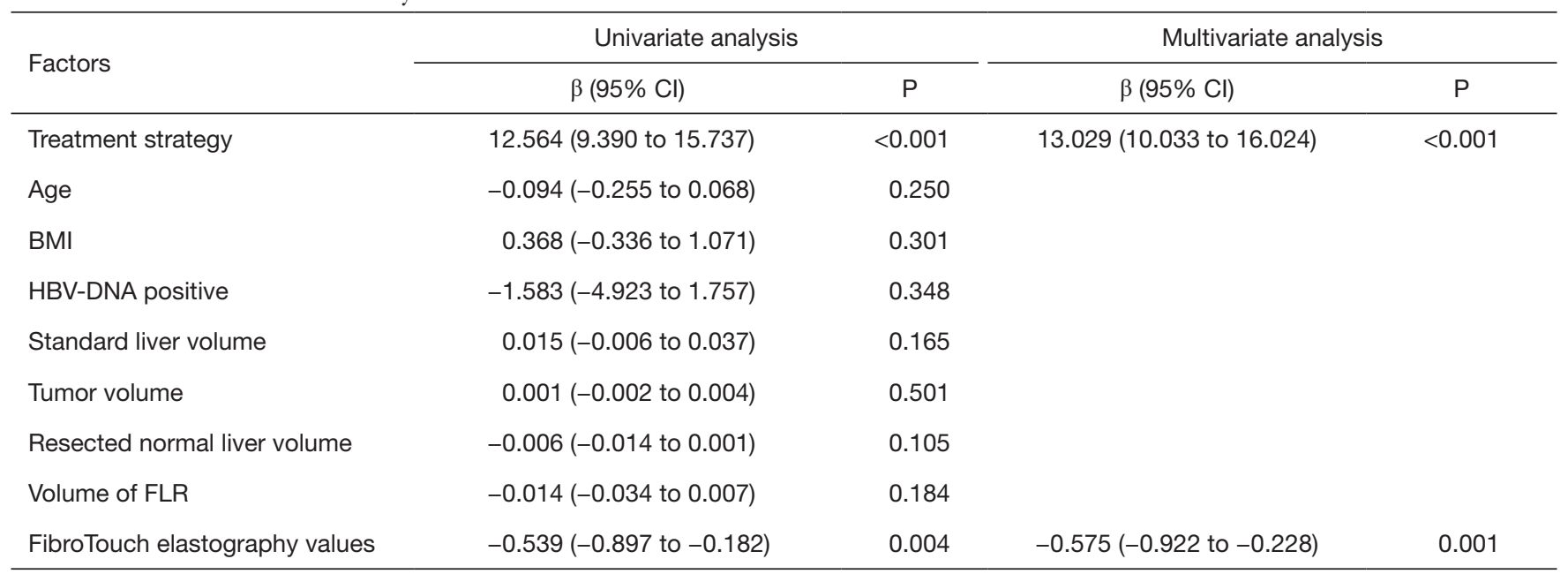

procedure, and the stage- 2 hepatectomy in the 2 groups are shown in Table 2. There were no significant differences between the 2 groups, except after the stage- 2 hepatectomy in the incidences of postoperative complication $\geq$ grade IIIa. Major postoperative complications developed in 20 of 37 patients $(54.1 \%)$ in the ALPPS group and 5 of 25 patients $(20.0 \%)$ in the TACE + PVE group (risk ratio 2.70, 95\% CI: $1.17-6.25, \mathrm{P}=0.007)$. All the postoperative complications were managed with conservative treatments, percutaneous drainage or reoperation. Two patients each in the ALPPS and TACE + PVE groups died of PHLF within 90 days of surgery. The liver function of the other patients with PHLF recovered fully, except in one patient who had persistently low prealbumin and cholinesterase, but normal total bilirubin and international normalized ratio levels.

There were no R1/R2 resections, and no viral reactivation on follow-up. The causes of 90-day mortality, and the treatments of HCC recurrence in the 2 groups are shown in Table 2. The comparison of postoperative pathologies between the 2 groups are shown in Table S1.

Median follow-up for survival was 42 months (IQR $38-45)$. The three-year OS rate of the ALPPS group (65.8\%) (95\% CI: 50.7-80.9) was significantly better than the TACE + PVE group (42.1\%) (95\% CI: 26.4-57.8) (HR 0.50, 95\% CI: 0.26-0.98, two-sided $\mathrm{P}=0.036$ ) (Figure $3 A$ ). Cox regression analyses showed that treatment with ALPPS (HR 0.34, 95\% CI: 0.16-0.74, $\mathrm{P}=0.006$ ), small tumor (HR 1.00, 95\% CI: $1.00-1.00, \mathrm{P}=0.0028$ ), single tumor (HR 11.83, 95\% CI: 3.98-35.15, $\mathrm{P}<0.001)$ and absence of severe liver fibrosis (HR 2.49, 95\% CI: 1.22-5.10, $\mathrm{P}=0.012$ ) were significant good risk factors of OS. There was no significant difference in the OS rates between patients who underwent tumor resection in the 2 groups of patients (HR 0.80, 95\% CI: 0.35-1.83, two-sided $\mathrm{P}=0.595$ ) (Figure 3B). However, there was a significant difference in the OS rates between patients who had or had not undergone liver resection in the TACE + PVE group (HR 4.45, 95\% CI: 1.85-10.68, two-sided $\mathrm{P}<0.001$ ) (Figure 3C). There was no significant difference in the disease-free survival (DFS) rates between patients who underwent tumor resection in the 2 groups of patients (HR 0.94, 95\% CI: 0.47-1.91, two-sided P=0.869). The three-year DFS rates of the 37 patients in ALPPS groups versus the 25 patients in TACE + PVE groups were $48.6 \%$ (95\% CI: $32.5-64.8$ ) versus $48 \%$ (95\% CI: $28.4-$ 67.6), respectively.

\section{Discussion}

Surgeons who support ALPPS believe it significantly increases the rate of liver resection $(9,31,32)$. Surgeons who object to it mainly because of its high morbidity and mortality rates (33). After more than a decade of development, complications and mortality rates of ALPPS have decreased through improvements in technology and strict selection of patients $(34,35)$. However, most reports on ALPPS are for colorectal liver metastases. Reports on HCC are rare $(36,37)$. While the former condition usually involves multiple secondary tumors in a normal liver, the latter usually involves a large primary tumor arising from a liver with a viral hepatitis background. Recent reports indicated that cirrhosis affects the rate of increase in volume of FLR and liver functional recovery after ALPPS. Whether 

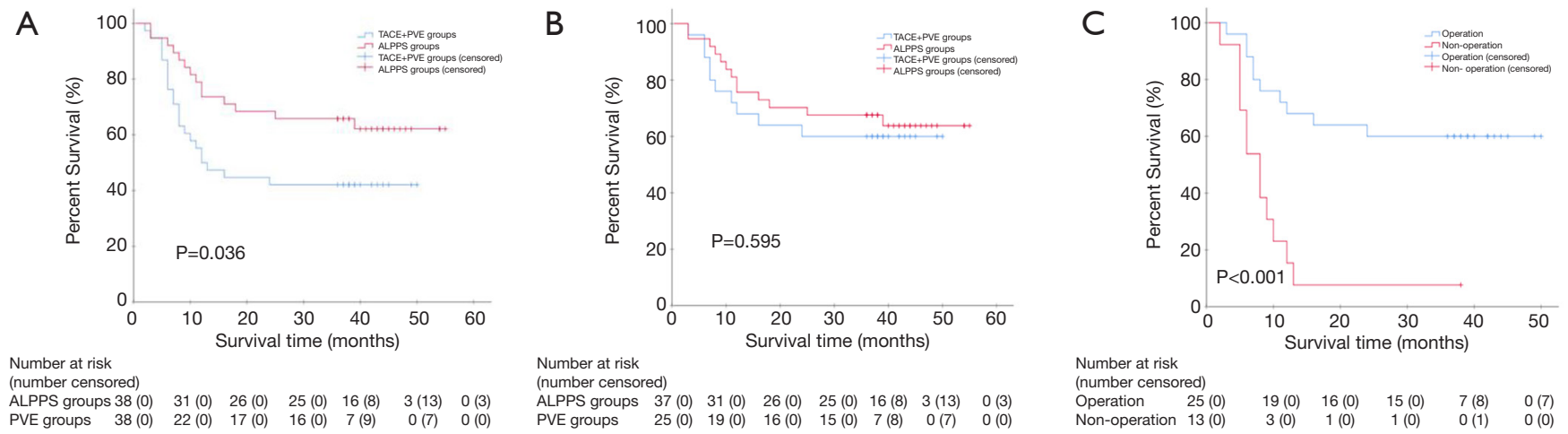

Figure 3 Overall survivals between different groups. (A) Comparison of overall survivals between all the patients (including the patients with or without tumor resection) in 2 groups; (B) comparison of overall survivals between the patients who had undergone stage-2 tumor resection in 2 groups; (C) comparison of overall survivals between the patients with and without tumor resection in PVE groups. PVE, portal vein embolization; TACE, transcatheter arterial chemoembolization; ALPPS, associating liver partition and portal vein ligation for staged hepatectomy.

ALPPS can be used in patients with primary or metastatic liver cancers to replace PVE is not known.

Our results suggested that ALPPS promoted adequate increase in volume of FLR even in HBV-related HCC patients with hepatic fibrosis. However, for patients with more severe cirrhosis, the growth rate and growth volume of FLR were limited and could not meet the criteria for stage- 2 resection in one patient. Our study also showed ALPPS to be better than TACE + PVE in that: (I) the interval between the stage 1 and 2 operations was significantly shorter. (II) The tumor resection rate was significantly higher. (III) The waiting time for the stage-2 operation was significantly shorter. All these made the resection rate of stage-2 hepatectomy significantly higher in the ALPPS group, making the three-year OS rate to become significantly better. However, if a stage-2 hepatectomy was successfully carried out, there was no significant difference in the OS rates between the 2 groups of patients. In the PVE group, the OS rate was significantly better in those patients who were able to undergo stage-2 hepatectomy. The incidence of major complications $(\geq$ IIIa) in the ALPPS group in our study, like other reported studies, was significantly higher than the PVE group $(9,33,38,39)$. There was no significant difference in the 90 -day mortality rates between the two groups. Reducing postoperative complication and mortality rates could be achieved by stringent patient selection and improvements in surgical techniques, such $3 \mathrm{D}$ visualization technique which can preoperatively show the 3D anatomical structures of the major intrahepatic vessels and anatomic variations.
Recent researches suggested that PVE with small spherical particles results in improved liver hypertrophy and resection rates compared with larger, nonspherical particles (40). Furthermore, as N-Butyl Cyanoacrylate (NBCA) can completely embolize the terminal branches of portal vein and the portal venous communicating branches, increase in volume of FLR has been reported to be fast enough to allow in a significant proportion of patients for stage-2 hepatectomy before tumor progression (41).

Our results showed portal hypertension reduced the rate of increase in FLR volume and stage-2 hepatectomy. The KGR was negatively correlated with the severity of liver fibrosis/cirrhosis. These findings suggested that selecting patients with severe cirrhosis and severe portal hypertension to undergo either ALPPS or PVE should be done very cautiously.

Our study also found increase in liver volume after ALPPS or PVE was not necessarily accompanied by synchronous recovery of full liver function. The prealbumin and cholinesterase levels were low in one patient, suggesting that the liver synthetic function had not fully recovered (42). To determine whether stage- 2 hepatectomy should be performed, a comprehensive judgement should be made by combining the FLR, indocyanine green clearance function test, prealbumin and cholinesterase levels and $99 \mathrm{mTc}-$ galactosyl serum albumin scintigraphy $(43,44)$.

This study had limitations. First, gelatin sponges and steel coils were used to embolize the portal vein. Second, whether the results of this study from China could be applied to HCC of other etiologies in other parts of the 
world is not known. Third, ALPPS was compared with TACE + PVE, but not with PVE only. TACE was given to slow down tumor progression during the long wait for hepatectomy as recommended by a published article (45). However, the rate of liver hypertrophy of FLR could be affected by TACE. Fourth, the patients failed to undergo hepatectomy in the TACE + PVE groups due to insufficient FLR didn't receive rescue ALPPS, which may increase the tumor resection rates in the TACE + PVE groups according to ITT.

\section{Conclusions}

The study showed ALPPS resulted in significantly better intermediate-term OS outcomes, at the cost of a significantly higher perioperative morbidity rate compared with TACE + PVE in patients who had initially unresectable HBV-related HCC.

\section{Acknowledgments}

Funding: This study was funded by the Science Fund for Creative Research Groups, NSFC, China (81521091); the State Key Infection Disease Project of China (2018ZX10732202-002-005) and the National Human Genetic Resources Sharing Service Platform (2005DKA21300).

\section{Footnote}

Conflicts of Interest: All authors have completed the ICMJE uniform disclosure form (available at https://hbsn.amegroups. com/article/view/10.21037/hbsn-20-264/coif). WPZ reports grants from NSFC, China, grants from State Key Infection Disease Project of China, grants from National Human Genetic Resources Sharing Service Platform, during the conduct of the study. The other authors have no conflicts of interest to declare.

Ethical Statement: The authors are accountable for all aspects of the work in ensuring that questions related to the accuracy or integrity of any part of the work are appropriately investigated and resolved. The study was conducted in accordance with the Declaration of Helsinki (as revised in 2013). The study was approved by the Ethics Committee of the Eastern Hepatobiliary Hospital (approval No. EHBHKY2014-03-019). A written informed consent was obtained from all the patients for their data to be used for research.

Open Access Statement: This is an Open Access article distributed in accordance with the Creative Commons Attribution-NonCommercial-NoDerivs 4.0 International License (CC BY-NC-ND 4.0), which permits the noncommercial replication and distribution of the article with the strict proviso that no changes or edits are made and the original work is properly cited (including links to both the formal publication through the relevant DOI and the license). See: https://creativecommons.org/licenses/by-nc-nd/4.0/.

\section{References}

1. Ozakyol A. Global Epidemiology of Hepatocellular Carcinoma (HCC Epidemiology). J Gastrointest Cancer 2017;48:238-40.

2. Kawaguchi Y, Honda G, Endo I, et al. Current Technical Issues for Surgery of Primary Liver Cancer. Liver Cancer 2016;6:51-8.

3. She WH. Strategies to increase the resectability of hepatocellular carcinoma. World J Hepatol 2015;7:2147.

4. Kishi Y, Abdalla EK, Chun YS, et al. Three hundred and one consecutive extended right hepatectomies: evaluation of outcome based on systematic liver volumetry. Ann Surg 2009;250:540-8.

5. van Mierlo KMC, Schaap FG, Dejong CHC, et al. Liver resection for cancer: New developments in prediction, prevention and management of postresectional liver failure. J Hepatol 2016;65:1217-31.

6. Makuuchi M, Thai BL, Takayasu K, et al. Preoperative portal embolization to increase safety of major hepatectomy for hilar bile duct carcinoma: a preliminary report. Surgery 1990;107:521-7.

7. Clavien PA, Petrowsky H, DeOliveira ML, et al. Strategies for safer liver surgery and partial liver transplantation. $\mathrm{N}$ Engl J Med 2007;356:1545-59.

8. Kianmanesh R, Farges O, Abdalla EK, et al. Right portal vein ligation: a new planned two-step all-surgical approach for complete resection of primary gastrointestinal tumors with multiple bilateral liver metastases. J Am Coll Surg 2003;197:164-70.

9. Schadde E, Ardiles V, Slankamenac K, et al. ALPPS Offers a Better Chance of Complete Resection in Patients with Primarily Unresectable Liver Tumors Compared with Conventional-Staged Hepatectomies: Results of a Multicenter Analysis. World J Surg 2014;38:1510-9.

10. de Santibañes E, Clavien PA. Playing Play-Doh to Prevent 
Postoperative Liver Failure: The "ALPPS" approach. Ann Surg 2012;255:415-7.

11. Zhang GQ, Zhang ZW, Lau WY, et al. Associating liver partition and portal vein ligation for staged hepatectomy (ALPPS): A new strategy to increase resectability in liver surgery. Int J Surg 2014;12:437-41.

12. Lau WY, Lai EC, Lau SH. Associating liver partition and portal vein ligation for staged hepatectomy: the current role and development. Hepatobiliary Pancreat Dis Int 2017;16:17-26.

13. Alvarez FA, Ardiles V, Sanchez Claria R, et al. Associating liver partition and portal vein ligation for staged hepatectomy (ALPPS): tips and tricks. J Gastrointest Surg 2013;17:814-21.

14. Schnitzbauer AA, Lang SA, Goessmann H, et al. Right Portal Vein Ligation Combined With In Situ Splitting Induces Rapid Left Lateral Liver Lobe Hypertrophy Enabling 2-Staged Extended Right Hepatic Resection in Small-for-Size Settings. Ann Surg 2012;255:405-14.

15. Abdalla EK, Adam R, Bilchik AJ, et al. Improving Resectability of Hepatic Colorectal Metastases: Expert Consensus Statement. Ann Surg Oncol 2006;13:1271-80.

16. Truant S, Oberlin O, Sergent G, et al. Remnant Liver Volume to Body Weight Ratio $\geq 0.5 \%$ : A New Cut-Off to Estimate Postoperative Risks after Extended Resection in Noncirrhotic Liver. J Am Coll Surg 2007;204:22-33.

17. Kiuchi T, Kasahara M, Uryuhara K, et al. Impact of graft size mismatching on graft prognosis in liver transplantation from living donors. Transplantation 1999;67:321-7.

18. Urata K, Kawasaki S, Matsunami H, et al. Calculation of child and adult standard liver volume for liver transplantation. Hepatology 1995;21:1317-21.

19. Bruix J, Reig M, Sherman M. Evidence-Based Diagnosis, Staging, and Treatment of Patients With Hepatocellular Carcinoma. Gastroenterology 2016;150:835-53.

20. de Franchis R, Baveno VI Faculty. Expanding consensus in portal hypertension: Report of the Baveno VI Consensus Workshop: Stratifying risk and individualizing care for portal hypertension. J Hepatol 2015;63:743-52.

21. Augustin S, Millán L, González A, et al. Detection of early portal hypertension with routine data and liver stiffness in patients with asymptomatic liver disease: A prospective study. J Hepatol 2014;60:561-9.

22. Aoki T. Sequential Preoperative Arterial and Portal Venous Embolizations in Patients With Hepatocellular Carcinoma. Arch Surg 2004;139:766-74.

23. Sarin SK, Kumar M, Lau GK, et al. Asian-Pacific clinical practice guidelines on the management of hepatitis B: a
2015 update. Hepatol Int 2016;10:1-98.

24. Huang G, Li P, Lau WY, et al. Antiviral Therapy Reduces Hepatocellular Carcinoma Recurrence in Patients With Low HBV-DNA Levels: A Randomized Controlled Trial. Ann Surg 2018;268:943-54.

25. Shindoh J, Truty MJ, Aloia TA, et al. Kinetic Growth Rate after Portal Vein Embolization Predicts Posthepatectomy Outcomes: Toward Zero Liver-Related Mortality in Patients with Colorectal Liver Metastases and Small Future Liver Remnant. J Am Coll Surg 2013;216:201-9.

26. Sandström P, Røsok BI, Sparrelid E, et al. ALPPS Improves Resectability Compared With Conventional Two-stage Hepatectomy in Patients With Advanced Colorectal Liver Metastasis: Results From a Scandinavian Multicenter Randomized Controlled Trial (LIGRO Trial). Ann Surg 2018;267:833-40.

27. Foucher J. Diagnosis of cirrhosis by transient elastography (FibroScan): a prospective study. Gut 2006;55:403-8.

28. Dindo D, Demartines N, Clavien PA. Classification of Surgical Complications: A New Proposal With Evaluation in a Cohort of 6336 Patients and Results of a Survey. Ann Surg 2004;240:205-13.

29. Rahbari NN, Garden OJ, Padbury R, et al. Posthepatectomy liver failure: a definition and grading by the International Study Group of Liver Surgery (ISGLS). Surgery 2011;149:713-24.

30. Bedossa P, Poynard T. An algorithm for the grading of activity in chronic hepatitis C. Hepatology 1996;24:289-93.

31. Jiao LR, Fajardo Puerta A, Gall T, et al. Rapid Induction of Liver Regeneration for Major Hepatectomy (REBIRTH):

A Randomized Controlled Trial of Portal Vein

Embolisation versus ALPPS Assisted with Radiofrequency. Cancers 2019;11:302.

32. Chan A, Zhang WY, Chok K, et al. ALPPS Versus Portal Vein Embolization for Hepatitis-related Hepatocellular Carcinoma: A Changing Paradigm in Modulation of Future Liver Remnant Before Major Hepatectomy. Ann Surg 2021;273:957-65.

33. Ratti F, Schadde E, Masetti M, et al. Strategies to Increase the Resectability of Patients with Colorectal Liver Metastases: A Multi-center Case-Match Analysis of ALPPS and Conventional Two-Stage Hepatectomy. Ann Surg Oncol 2015;22:1933-42.

34. Li J, Ewald F, Gulati A, et al. Associating liver partition and portal vein ligation for staged hepatectomy: From technical evolution to oncological benefit. World J Gastrointest Surg 2016;8:124-33. 
35. Linecker M, Björnsson B, Stavrou GA, et al. Risk Adjustment in ALPPS Is Associated With a Dramatic Decrease in Early Mortality and Morbidity: Ann Surg 2017;266:779-86.

36. Wang Z, Peng Y, Hu J, et al. Associating Liver Partition and Portal Vein Ligation for Staged Hepatectomy for Unresectable Hepatitis B Virus-related Hepatocellular Carcinoma: A Single Center Study of 45 Patients. Ann Surg 2020;271:534-41.

37. Chan ACY, Chok K, Dai JWC, et al. Impact of split completeness on future liver remnant hypertrophy in associating liver partition and portal vein ligation for staged hepatectomy (ALPPS) in hepatocellular carcinoma: Complete-ALPPS versus partial-ALPPS. Surgery 2017;161:357-64.

38. Shindoh J, Vauthey JN, Zimmitti G, et al. Analysis of the Efficacy of Portal Vein Embolization for Patients with Extensive Liver Malignancy and Very Low Future Liver Remnant Volume, Including a Comparison with the Associating Liver Partition with Portal Vein Ligation for Staged Hepatectomy Approach. J Am Coll Surg 2013;217:126-33.

39. Eshmuminov D, Raptis DA, Linecker M, et al. Metaanalysis of associating liver partition with portal vein ligation and portal vein occlusion for two-stage hepatectomy. Br J Surg 2016;103:1768-82.

40. Madoff DC, Abdalla EK, Gupta S, et al. Transhepatic Ipsilateral Right Portal Vein Embolization Extended

Cite this article as: Li PP, Huang G, Jia NY, Pan ZY, Liu H, Yang Y, He CJ, Lau WY, Yang YF, Zhou WP. Associating liver partition and portal vein ligation for staged hepatectomy versus sequential transarterial chemoembolization and portal vein embolization in staged hepatectomy for HBV-related hepatocellular carcinoma: a randomized comparative study. HepatoBiliary Surg Nutr 2022;11(1):38-51. doi: 10.21037/hbsn20-264 to Segment IV: Improving Hypertrophy and Resection Outcomes with Spherical Particles and Coils. J Vasc Interv Radiol 2005;16:215-25.

41. Luz JHM, Luz PM, Bilhim T, et al. Portal vein embolization with n-butyl-cyanoacrylate through an ipsilateral approach before major hepatectomy: single center analysis of 50 consecutive patients. Cancer Imaging 2017;17:25.

42. Matsuo K, Murakami T, Kawaguchi D, et al. Histologic features after surgery associating liver partition and portal vein ligation for staged hepatectomy versus those after hepatectomy with portal vein embolization. Surgery 2016;159:1289-98.

43. Truant S, Baillet C, Deshorgue AC, et al. Drop of Total Liver Function in the Interstages of the New Associating Liver Partition and Portal Vein Ligation for Staged Hepatectomy Technique: Analysis of the "Auxiliary Liver" by HIDA Scintigraphy. Ann Surg 2016;263:e33-4.

44. Kwon AH, Matsui Y, Ha-Kawa SK, et al. Functional hepatic volume measured by technetium-99m-galactosylhuman serum albumin liver scintigraphy: comparison between hepatocyte volume and liver volume by computed tomography. Am J Gastroenterol 2001;96:541-6.

45. Ronot M, Cauchy F, Gregoli B, et al. Sequential transarterial chemoembolization and portal vein embolization before resection is a valid oncological strategy for unilobar hepatocellular carcinoma regardless of the tumor burden. HPB 2016;18:684-90. 
Supplementary

Table S1 Comparison of pathologic results between two groups

\begin{tabular}{lccc}
\hline Parameters & ALPPS group $(\mathrm{n}=37)$ & PVE group $(\mathrm{n}=25)$ & P value \\
\hline Tumor type & & & \\
Hepatocellular carcinoma, $\mathrm{n}(\%)$ & $37(100 \%)$ & $25(100 \%)$ & 0.258 \\
Maximum tumor size (cm) & $9.2(3.1-15.4)$ & $8.5(3.6-14.4)$ & 0.960 \\
Multiple tumor, $\mathrm{n}(\%)$ & $18(48.6 \%)$ & $12(48.0 \%)$ & 0.826 \\
Presence of cirrhosis, $\mathrm{n}(\%)$ & $8(21.6 \%)$ & $6(24.0 \%)$ & 0.796 \\
Microvascular invasion, $\mathrm{n}(\%)$ & $18(48.6 \%)$ & $13(52.0 \%)$ & 0.916 \\
Capsule integrity, $\mathrm{n}(\%)$ & $7(18.9 \%)$ & $5(20.0 \%)$ & \\
\hline
\end{tabular}

